\title{
Estado y prospectiva de los PRAU (Proyectos Ambientales Universitarios) en las universidades presentes en la ciudad de Montería
}

State and Prospective of the PRAU (Proyectos Ambientales Universitarios) in the Universities present in the city of Monteria

Carlos Emilio Atencia Benítez

Estudiante investigador, Universidad Santo Tomás programa de Administración ambiental y de los recursos naturales. Proyecto de grado, para optar al título de grado en Administración ambiental y de los recursos naturales.

Correo electrónico: carlosatencia@ustadistancia.edu.co

Igor Julio Peniche Villadiego

Docente universitario - investigador. Universidad Santo Tomás programa de Administración ambiental y de los recursos naturales. Director del proyecto de grado.

Alonso de Jesús Agudelo Nisperuza

Docente universitario - investigador. Universidad Santo Tomás programa de Administración ambiental y de los recursos naturales.

Correo electrónico: alonsoagudelo@ustadistancia.edu.co 


\section{Resumen}

En el presente trabajo se presentará un análisis del estado y hacia donde se focalizan los proyectos ambientales universitarios PRAU-, en las universidades que ofrecen sus servicios educativos en la ciudad de Montería, y poder establecer cuál es su contribución en la resolución de problemáticas ambientales de su entorno y en especial de la ciudad. Las visitas a los establecimientos universitarios nos permitieron acercarnos, al enfoque de estos como estrategia de mitigación de dichas problemáticas, a través del contacto directo con los profesionales encargados de la dirección; de igual manera el acercamiento a la población estudiantil y evaluar su percepción, lo cual es contrastado con la información de campo de otros grupos de interés como juntas de acción locales, organizaciones no gubernamentales, así como instituciones públicas y privadas que puedan aportar conocimiento a esta investigación. El estudio nos presentará una radiografía acerca de las dinámicas de estos proyectos al interior de las universidades y como son vistos por las comunidades como estrategias de solución a sus problemáticas ambientales. De igual manera, se espera que la investigación genere una nueva mirada hacia estas estrategias, como eje articulador de políticas de educación ambiental, a través del empoderamiento y la proyección social de las universidades.

Palabras clave: Proyectos Ambientales Universitarios (PRAU), educación ambiental, política ambiental, problemática ambiental, grupos de interés.
In the present work an analysis of the state and towards where the PRAUS university environmental projects are focused will be presented, in the universities that offer their educational services in the city of Monteria and to be able to establish what is their contribution in the resolution of environmental problems of their environment and especially the city. The visits to the university establishments allowed us to approach the approach of these as a mitigation strategy of these problems, through direct contact with the professionals in charge of the management, in the same way the enclosure to the student population and to evaluate their perception, all this will be contrasted with the field information of other stakeholders such as local action boards, non-governmental organizations, public and private institutions that can contribute knowledge to this research. The study will present an x-ray about the dynamics of these projects within the universities and how they are viewed by the communities as strategies to solve their environmental problems. In the same way, this originates a new look towards these strategies, as a milestone of environmental education policy, through the empowerment and social projection of the universities.

Keywords: University Environmental

Projects, environmental education, environmental policy. 


\section{Introducción}

La educación ambiental esta llamada a ser uno de los pilares fundamentales de una formación integral, en tanto que propende por la armonía entre el ser humano y su entorno natural, ya que no se puede concebir al hombre científico sin una dimensión humana que le permita potenciar no solo su dimensión cognitiva, sino también la ética y la estética de su ser.

Desde esta perspectiva, las universidades juegan un papel importante teniendo en cuenta que deben ofrecer al país profesionales capaces de afrontar los nuevos retos que aparecen en su sociedad, en donde se puede percibir que los problemas ambientales son cada día más y mayores, y donde la academia debe hacer un aporte considerable propiciando espacios investigativos que apunten a la solución de dicha problemática.

Y es que esta preocupación por la conservación medioambiental ha despertado interés en todas las esferas de la sociedad, hasta tal punto que el mismo papa Francisco ha tomado la iniciativa de exponer en su encíclica un amplio tratado respecto al medio ambiente, expresando en él una profunda preocupación por el estado actual de lo que él denomina "la Casa Común".

“El desafío urgente de proteger nuestra casa común incluye la preocupación de unir a toda la familia humana en la búsqueda de un desarrollo sostenible e integral, pues sabemos que las cosas pueden cambiar. El Creador no nos abandona, nunca hizo marcha atrás en su proyecto de amor, no se arrepiente de habernos creado. La huma $\neg$ nidad aún posee la capa- cidad de colaborar para construir nuestra casa común. Deseo reconocer, alentar y dar las gracias a todos los que, en los más variados sectores de la actividad humana, es $\neg$ tán trabajando para garantizar la protección de la casa que compartimos. Merecen una gratitud es $\neg$ pecial quienes luchan con vigor para resolver las consecuencias dramáticas de la degradación am?biental en las vidas de los más pobres del mundo" (Francisco, 2015).

En cuanto a la prospectiva, ésta nos permite visualizar el futuro y actuar en el presente. La prospectiva no pretende adivinar la ocurrencia de un hecho, sino que busca reducir notablemente la incertidumbre en torno a su ocurrencia, con sus potentes faros anticipatorios iluminando con ello las acciones que se deben tomar en el presente.

La prospectiva, por lo tanto, no sólo pretende conocer el futuro de manera anticipada, sino fundamentalmente diseñarlo y construirlo colectivamente en forma participativa, lo cual aplicado al caso al que se hace referencia en el presente tratado, se traduce en la toma de conciencia y decisiones en el presente, con el propósito de disminuir los riesgos y afectaciones futuras en cuanto a lo ambiental, teniendo como norte la concepción de un desarrollo sostenible, en el que se satisfagan las necesidades de la presente generación y se garantice la satisfacción de las necesidades de las generaciones futuras.

Lo que se pretende en la siguiente investigación, es establecer cuál es el estado de actual de los PRAU en las diferentes universidades presentes en la ciudad de Montería, si existen; y cuál es la prospectiva de los mismos, es decir hacia donde apuntan y si guardan el carácter 
interinstitucional que estos deben tener. De no existir, plantear de manera colectiva y participativa la implementación de éstos, con el fin de propiciar espacios investigativos en temas ambientales, que apunten a la solución de las diferentes problemáticas que este aspecto sugiere.

En ese orden de ideas, en la presente investigación se hará hincapié en aspectos destacados pertinentes a los PRAU como son el cumplimiento normativo que implica para las universidades la implementación de los mismos, la interdisciplinariedad de los proyectos, la institucionalización de éstos, sus principios rectores y la responsabilidad de la comunidad educativa ante ellos.

\section{Materiales y métodos}

El proceso a través del cual se realizará la presente propuesta de investigación, se plantea a partir de desarrollar las siguientes etapas: consulta o revisión de PRAU, levantamiento, procesamiento, análisis y síntesis de información de los Proyectos Ambientales Universitarios. Cabe destacar que esta investigación se enmarca dentro de lo empírico-analítico, lo cual permite investigar la realidad como un todo constituido por dimensiones o variables, analizables y cuantificables.

En la ciudad de Montería hacen presencia de manera formal 12 universidades, con un predominio de las de carácter privado, pues 11 de estas corresponden a este sector y solo una es de carácter público.

La recolección de la información de campo y la técnica documental, consistió en la aplica- ción de encuestas, las cuales se diseñaron, enfocadas en tres grupos de interés: estudiantes, docentes y directivos y grupos afines.

Para conocer el tamaño de la muestra, cuenta partir de que este es un diseño de muestreo aleatorio simple (MAS), en la que se tomaron muestras representativas, teniendo en cuenta la homogeneidad presentada en las universidades, se realizaron encuestas en cada una de las instituciones, subdivididas y enfocadas a los estudiantes (20), directivos y docentes (4).

En cuanto al tamaño de la muestra, para los grupos afines o de interés, se utilizó el muestreo no probabilístico o por conveniencia, el cual consiste en seleccionar una muestra de la población por el hecho de ser accesible al encuestador. Es decir, los individuos empleados en la investigación se seleccionan porque están fácilmente disponibles, no porque hayan sido seleccionados mediante un criterio estadístico, que para el presente caso fue tenido en cuenta un numero de 20 grupos que reúnen las características antes mencionadas.

\section{Resultado y análisis}

La situación problémica que conllevó a la detección de la situación actual de los PRAU en las universidades presentes en la ciudad de Montería, nos planteó como primer interrogante ¿Sabe usted que es un PRAU?, para esto analizamos el siguiente gráfico. 
Gráfico 1. Concepto de PRAU para los estudiantes.

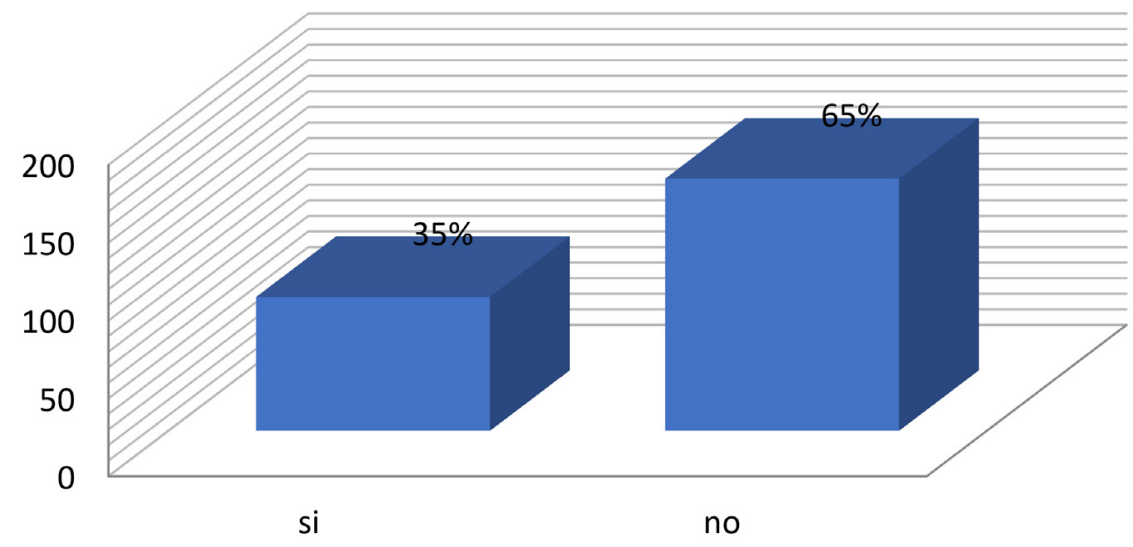

Fuente. Autores (2018).

El 65\% de la población estudiantil encuestada no tiene conocimiento de lo que es un PRAU, y solo un 35\% de la población encuestada tiene algún grado de conocimiento, lo que evidencia que este es un tema poco común para la comunidad universitaria y que el aspecto ambiental debería ser un tema transversal a todos los programas universitarios, de modo que no solo aquellos que enfoquen sus estudios a programas afines con lo ambiental. De la misma manera al ser consultados los directivos y docentes, el $42 \%$ manifiesta no tener conocimiento de esta temática y menos de la existencia de un PRAU al interior de su universidad.

Todo esto se refuerza a través del ítem 7 de la encuesta para estudiantes en la pregunta: ¿Cuál cree usted que es la finalidad de los PRAU? se pudo establecer que el $70 \%$ de los estudiantes encuestados no tienen claro cuál es la finalidad de los PRAU, solo el 30\% del total de encuestados dio una respuesta cohe- rente con la finalidad de estos proyectos, dejando ver que en las universidades este no es un tema de gran dominio.

En lo que respecta a los diferentes grupos de interés encuestados respecto a la misma temática, sobre si tenía conocimiento de lo que es un PRAU, se encontró que los porcentajes son muy similares, pues un $60 \%$ manifestó no saber que es un PRAU, en tanto que el restante $40 \%$ dijo tener algún tipo de idea de lo que era esto. Llama la atención que en comparación con los estudiantes encuestados, el porcentaje de conocimiento de lo que es un PRAU es menor que el de estos grupos de interés, es decir, tienen más conocimiento de esto dichos grupos que los estudiantes mismos.

Frente a la consulta a los diferentes grupos de interés respecto de la manera cómo estos grupos están dispuestos a participar ¿De qué manera participaría su organización en la solución a la problemática ambiental que les 
afecta?, se puede notar que existe cierto grado de heterogeneidad en los porcentajes, lo cual quiere decir que a pesar de la disposición de los grupos para participar, existe un alto grado de desorientación al respecto, pues a pesar de estar dispuestos a participar no hay un punto común hacia donde apunte su disposición de trabajo. Sin embargo, este es un buen punto de partida, ya que correspondería a la academia realizar un buen trabajo de orientación que permita canalizar todo este potencial para que confluya en un punto común para lograr el máximo de provecho de este potencial.

En la implementación de estos proyectos hay muchos factores en juego que impiden que los mismos se lleven a cabo, esta verdad se pudo establecer a través de la siguiente pregunta ¿porque no se están implementando los PRAU en las universidades de la ciudad de Montería? se pudo establecer que entre los factores negativos que dificultan la puesta en marcha de esta clase de iniciativa, está en un mayor porcentaje la falta de compromiso de la comunidad universitaria con un $50 \%$ de las causas de la no implementación de éstos, seguida por la falta de gestión con un 33\% y la falta de recursos económicos con un 17\%, tal y como lo muestra la siguiente tabla que responde a la pregunta: ¿Qué dificultades limitan el desarrollo del PRAU en su institución?, de la encuesta planteada para docentes y directivos.

Tabla 1. Dificultades que limitan el desarrollo de los PRAU en la ciudad de Montería

\begin{tabular}{|l|l|l|}
\hline \multicolumn{2}{|c|}{ Tabla de frecuencia } \\
\hline Respuestas & $n_{i}$ & $f_{i}$ \\
\hline Renuencia de los docentes a participar & & \\
\hline Falta de compromiso de la comunidad para liderar procesos & 22 & $50 \%$ \\
\hline Recursos humanos & & \\
\hline Recursos económicos & 8 & $17 \%$ \\
\hline Falta de gestión & 14 & $33 \%$ \\
\hline
\end{tabular}

Fuente. Autores (2018)

Ante el interrogante preguntado a directivos y docentes ¿Estados de los PRAU en las universidades de la ciudad de Montería? y que se correlacionó con los resultados de los estudiantes, desde la perspectiva de las directivas universitarias, encontramos que $58 \%$ de las instituciones encuestadas tienen en alguna fase el Proyecto Ambiental Universitario. De este porcentaje la investigación muestra que un $50 \%$ se encuentra en etapa de identificación de problemas, un $25 \%$ en formulación, un $17 \%$ en implementación y solo un $8 \%$ se encuentra en la fase de evaluación y seguimiento. Con- cretamente lo que se quiere señalar en el párrafo anterior, es que ese $58 \%$ representa 7 de las 12 universidades encuestadas, de las cuales 3 se encuentran en fase de identificación; 2 en fase de implementación; 1 en fase de formulación y 1 en fase de evaluación y seguimiento.

Acerca de la consulta referente a ¿Acompañamiento de expertos en la gestión de la interdisciplinariedad de los PRAU en la ciudad de Montería? A través del estudio se quiso establecer si hubo algún acompañamiento por parte de algún grupo de expertos para la gestión de 
la interdisciplinariedad en la elaboración de dicho proyecto, entendiendo que ésta es un factor fundamental a la hora de elaborar los PRAU, pues no se pueden concebir éstos sin la conformación de un grupo interdisciplinario que maneje adecuadamente cada aspecto del proyecto, o en su defecto los distintos aspectos que serían susceptibles de abordar a través de un proyecto ambiental universitario.

El estudio muestra en una medida porcentual a través del ítem 6 de la encuesta para directivos docentes, que en el $67 \%$ de estas instituciones encuestadas han tenido expertos en la gestión de la interdisciplinariedad de los proyectos ambientales, y que un 33\% no han tenido este acompañamiento, es decir, fijándose en las preguntas anteriores en las que las instituciones tienen grupos de trabajos en estos proyectos, un porcentaje considerable ha gestionado o hecho posible que se de este acompañamiento en estas instituciones pero que sin embargo este aspecto no abarca en un $100 \%$ esta condición para que se de este tipo de proyectos de manera adecuada.

\section{Conclusiones}

A través de este estudio se pudo establecer:

En lo relacionado con el diagnóstico actual de los PRAU en la ciudad de Montería, se pudo establecer, de acuerdo con la información arrojada por la encuesta para directivos y docentes, que solo en un $58 \%$ de las instituciones se han implementado estos proyectos, es decir solo un poco más de la mitad de universidades lo han realizado. Con esto queda de manifiesto que existe un déficit del $42 \%$ de universidades que no están implementando esta iniciativa, incurriendo en un desacato de la normativa que indica la obligatoriedad de estos proyectos.

El 58\% a que se hace referencia, de las universidades que tienen en alguna fase su PRAU, representa a 7 universidades del total de 12 a las que se le aplicó la encuesta, de las cuales solo dos han implementado el proyecto, una se encuentra en fase de evaluación y seguimiento y las otras cuatro restantes se encuentran en fase de identificación de problemas. Esto quiere decir que en materia de aplicación de estos proyectos las universidades de esta ciudad tienen un gran atraso ya que lo ideal sería que por lo menos que estas siete estén en una fase más avanzada. Así mismo, en lo relacionado con el estado de implementación en que se encuentran los PRAUS en esta ciudad, el análisis muestra que no existe una concordancia entre lo que dicen los alumnos y lo que manifiestan los docentes y directivos, pues mientras que, de acuerdo con los primeros, la implementación se da en un $20 \%$, los segundos dicen que tal implementación se da en un 58\% generando un espacio de debate al respecto.

El estudio demuestra que existen muchas falencias en la forma como se han formulado los Proyectos Ambientales Universitarios en las diferentes instituciones de educación superior de esta ciudad, pues no son incluyentes ya que algunos actores que juegan un papel importante en la formulación de estos proyectos no han sido tenidos en cuenta, de esta manera se puede concluir que su concepción no ha sido de una manera integral en donde todos los grupos de interés tengan una participación activa. 
Dada la amplitud que puede abarcar un proyecto ambiental universitario se puede afirmar que la temática que abordan los PRAU consultados a través del estudio, apuntan a la resolución de problemas puntuales que se pueden abordar desde la perspectiva ambiental, sin embargo se ha dejado de lado el componente social, es decir, existe la necesidad de plantear proyectos enfocados al fortalecimiento del tejido social, entendiendo que este es un factor importante, máxime cuando estamos ad portas de la firma de un acuerdo de paz y en donde el fortalecimiento de la sociedades en este aspecto va a ser determinante para el éxito de los acuerdos pactados.

En una síntesis general, se pudo establecer que no hay un punto de congruencia entre la percepción de los estudiantes y la de los directivos docentes respecto al estado actual de los PRAU en las universidades de la ciudad de Montería, pues como ya se había manifestado, el estudio muestra que mientras los primeros consideran que apenas el $20 \%$ de las universidades lo está implementando; los segundos dicen que estos proyectos se están implementando en el 58\% de las universidades de dicha ciudad. Queda claro entonces que los PRAU no son una iniciativa que se esté implementando en el $100 \%$ de las universidades de la ciudad de Montería y que en este aspecto hay muchas cosas por hacer. Así las cosas, no hay una prospectiva clara respecto a los proyectos ambientales universitarios en esta ciudad, es decir no tienen un norte definido claramente hacia donde confluyan.

\section{Referencias}

Artieda, G. (1999). Educación ambiental: cuestiones básicas. Instituto Geográfico Vasco. Recuperado de: http://www.ingeba.org/ lurralde/lurranet/lur22/goran22/22ogran.htm

Benítez, M. (2014). Medio Ambiente. Recuperado de: prezi.com/vkznlcyppwvw/medio-ambiente

Cambio Climático. Org. (2010). Convención marco de la ONU sobre cambio climático. Recuperado de: http://www.cambioclimatico.org/tema/convencion-marco-naciones-unidascambio-climatico-cmnucc

Congreso de la República. (1994). Ley 115 de febrero 8 de 1994, por la cual se expide la ley general de educación. Recuperado de: https://www.mineducacion.gov.co/1621/ articles-85906_archivo_pdf.pdf

Dane. (2010). Proyección demográfica Montería. Enero 24, 2016, de Alcaldía de Montería Sitio web: www.dane.gov.co/index 64

Instituto Alexander Von Humboldt (2008). Educación ambiental y biodiversidad.

Francisco. Vaticano. (2015). Carta Encíclica LAUDATO SI del santo padre Francisco. Ciudad Vaticano: Tipografía Vaticana. Recuperado de: http://w2.vatican.va/content/ $\mathrm{dam} / \mathrm{francesco/pdf/encyclicals/docu-}$ ments/papa-francesco_20150524_enciclica-laudato-si_sp.pdf 
Ministerio de Educación (2012). Ley 1549 de julio 05 de 2012, Por medio de la cual se fortalece la institucionalización de la política nacional de educación ambiental y su incorporación efectiva en el desarrollo territorial. Recuperado de: http://www. suin-juriscol.gov.co/viewDocument.asp?ruta $=$ Leyes $/ 1683174$

Ministerio de Educación Nacional (1994). Decreto 1743 de 1994, Por el cual se instituye el Proyecto de Educación Ambiental para todos los niveles de educación formal, se fijan criterios para la promoción de la educación ambiental no formal e informal y se establecen los mecanismos de coordinación entre el Ministerio de Educación nacional y el Ministerio del Medio Ambiente. Recuperado de: http://www.minambiente. gov.co/images/BosquesBiodiversidadyServiciosEcosistemicos/pdf/Normativa/ Decretos/dec_1743_030894.pdf

Rojas, J. (2007). Proyectos Ambientales Universitarios- lineamientos teóricos. de Ministerio de Ambiente, Vivienda y Desarrollo Rural. Recuperado de: www.redcolombianafa.org/apc.../praus___documento_ de_trabajo

Peniche, I. (2014). Impactos de los PRAUen la construcción de valores sociales y ambientales en el municipio de Montería. Proyecto de investigación. Universidad Santo Tomás. Sede Montería.

Tamayo, M. (2008). El proceso de la investigación científica. $4^{\circ}$ edición. México D.F: Limusa.
Toro, J. \& Lowy, P. (2005). Educación ambiental: una visión de valores. Recuperado de: http://www.ingeba.org

Unesco. (1977). Conferencia Internacional de Educación Ambiental. 20/11/2015, Recuperado de: http://unesdoc.unesco.org

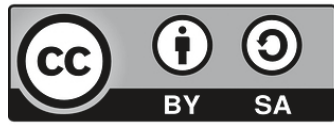

